\title{
Majorization involving the cyclic moving average
}

Tao Zhang ${ }^{1 *}$, Huan-Nan Shi ${ }^{2}$, Bo-Yan $\mathrm{Xi}^{3}$ and Alatancang Chen ${ }^{1}$

"Correspondence:

zhangtaocx@163.com

'School of Mathematical Sciences, Inner Mongolia University, Hohhot,

China

Full list of author information is available at the end of the article

\section{Abstract \\ We solve an open problem on some majorization inequalities involving the cyclic moving average.}

MSC: 26D15; 26E60; 26A51

Keywords: Inequality; Cyclic moving average

\section{Introduction}

We first recall two definitions.

Definition 1.1 ([1]) For fixed $n \geq 2$, let $x=\left(x_{1}, x_{2}, \ldots, x_{n}\right)$ and $y=\left(y_{1}, y_{2}, \ldots, y_{n}\right)$ be two $n$-tuples of real numbers.

(i) $x$ is said to be majorized by $y$ (in symbols, $x \prec y$ ) if

$$
\sum_{i=1}^{k} x_{[i]} \leq \sum_{i=1}^{k} y_{[i]} \quad \text { for } k=1,2, \ldots, n-1, \quad \text { and } \quad \sum_{i=1}^{n} x_{i}=\sum_{i=1}^{n} y_{i}
$$

where $x_{[1]} \geq \cdots \geq x_{[n]}$ and $y_{[1]} \geq \cdots \geq y_{[n]}$ are rearrangements of $x$ and $y$ in descending order.

(ii) Let $\Omega \subset \mathbb{R}^{n}$. A function $\varphi: \Omega \rightarrow \mathbb{R}$ is said to be a Schur-convex function (shortly, an $S$-convex function) if

$$
x \prec y \Rightarrow \varphi(x) \leq \varphi(y) .
$$

For example:

$$
\begin{aligned}
& a^{(2)}=\left(\frac{a_{1}+a_{2}}{2}, \frac{a_{2}+a_{3}}{2}, \ldots, \frac{a_{n}+a_{1}}{2}\right), \\
& a^{(3)}=\left(\frac{a_{1}+a_{2}+a_{3}}{3}, \frac{a_{2}+a_{3}+a_{4}}{3}, \ldots, \frac{a_{n}+a_{1}+a_{2}}{3}\right) .
\end{aligned}
$$

In 2006, I. Olkin, one of the authors of the book [1], wrote a letter to K. Z. Guan, referring to the following interesting question: is it true that

$$
a^{(k+1)} \prec a^{(k)}, \quad 1 \leq k \leq n-1 ?
$$

(c) The Author(s) 2018. This article is distributed under the terms of the Creative Commons Attribution 4.0 International License (http://creativecommons.org/licenses/by/4.0/), which permits unrestricted use, distribution, and reproduction in any medium, provided you give appropriate credit to the original author(s) and the source, provide a link to the Creative Commons license, and indicate if changes were made. 
However, a proof for $a^{(k+1)} \prec a^{(k)}$ remains elusive (see [1], p. 63).

In 2010, Shi [2] proved that (1) holds when $n=4, k=2$ and $n=5, k=3$. In this paper, we prove that (1) holds for any $n \geq 2$ and $1 \leq k \leq n-1$.

For any $1 \leq k \leq n$, let

$$
a_{[1]}^{(k)} \geq a_{[2]}^{(k)} \geq \cdots \geq a_{[n]}^{(k)}
$$

be the ordered component of the sequence $a_{1}^{(k)}, a_{2}^{(k)}, \ldots, a_{n}^{(k)}$. We denote

$$
S_{h}^{(k)}=\sum_{i=1}^{h} a_{[i]}^{(k)}, \quad 1 \leq h \leq n .
$$

\section{Lemmas and corollaries}

For proving our main results, we need the following lemmas.

Lemma 2.1 Let $n \geq 2$ and $1 \leq k \leq n-1$. Then

$$
\left\{\begin{array}{l}
a_{1}^{(k)} \geq a_{2}^{(k)} \geq \cdots \geq a_{n-k+1}^{(k)} \\
a_{n}^{(k)} \geq a_{n-1}^{(k)} \geq \cdots \geq a_{n-k+1}^{(k)} \\
a_{1}^{(k)} \geq a_{n}^{(k)}
\end{array}\right.
$$

Proof For any $1 \leq k \leq n-1$ and $1 \leq i \leq n$, we have

$$
k\left(a_{i}^{(k)}-a_{i+1}^{(k)}\right)=\left(a_{i}+a_{i+1}+\cdots+a_{i+k-1}\right)-\left(a_{i+1}+a_{i+2}+\cdots+a_{i+k}\right)=a_{i}-a_{i+k} .
$$

Note that $a_{1} \geq a_{2} \geq \cdots \geq a_{n}$, so we can induce that

(1) if $i+k \leq n$, that is, $1 \leq i \leq n-k$, then $a_{i} \geq a_{i+k}$. It follows that $a_{i}^{(k)} \geq a_{i+1}^{(k)}$.

(2) if $i+k>n$, that is, $n-k<i \leq n$, then $a_{i+k}=a_{n+(i+k-n)}=a_{i+k-n}$. Since $1 \leq k \leq n-1$, we have $n \geq i>i+k-n$. So $a_{i} \geq a_{i+k-n}=a_{i+k}$. It follows that $a_{i}^{(k)} \geq a_{i+1}^{(k)}$.

(3) $k\left(a_{1}^{(k)}-a_{n}^{(k)}\right)=\left(a_{1}+a_{2}+\cdots+a_{k}\right)-\left(a_{n}+a_{1}+\cdots+a_{k-1}\right)=a_{k}-a_{n} \geq 0$. Therefore (2) holds.

From the proof of Lemma 2.1 it is easy to deduce the following:

Corollary 2.2 Let $n \geq 2,1 \leq k \leq n-1$, and let $\sum_{i=0}^{-1} a_{n-i}^{(k)}=0$. For any $1 \leq h \leq n-1$, there exist $1 \leq h_{1} \leq n-k$ and $-1 \leq h_{2} \leq k-2$ such that $h=h_{1}+h_{2}+1$ and

$$
S_{h}^{(k)}=\sum_{i=1}^{h_{1}} a_{i}^{(k)}+\sum_{i=0}^{h_{2}} a_{n-i}^{(k)} .
$$

Lemma 2.3 Let $n \geq 4,2 \leq k \leq n-2$, and $0 \leq r \leq k-2$.

(i) If

$$
a_{2}^{(k)} \leq a_{n-r}^{(k)} \leq a_{1}^{(k)},
$$

then

$$
a_{2}^{(k+1)} \leq a_{n-r}^{(k+1)} \leq a_{1}^{(k+1)} .
$$


(ii) If

$$
a_{n-k+1}^{(k)} \leq a_{n-r}^{(k)} \leq a_{n-k}^{(k)}
$$

then

$$
a_{n-k}^{(k+1)} \leq a_{n-r-1}^{(k+1)} \leq a_{n-k-1}^{(k+1)}
$$

(iii) For $2 \leq m \leq n-k-1$, if

$$
a_{m+1}^{(k)} \leq a_{n-r}^{(k)} \leq a_{m}^{(k)}
$$

then

$$
a_{m+1}^{(k+1)} \leq a_{n-r}^{(k+1)}, \quad a_{n-r-1}^{(k+1)} \leq a_{m-1}^{(k+1)} .
$$

(iv) If

$$
a_{n}^{(k)} \leq a_{n-k}^{(k)}
$$

then

$$
a_{n-1}^{(k+1)} \leq a_{n-k-1}^{(k+1)}
$$

(v) For $2 \leq m \leq n-k-1$, if

$$
a_{n}^{(k)} \leq a_{m}^{(k)}
$$

then

$$
a_{n-1}^{(k+1)} \leq a_{m}^{(k+1)}
$$

(vi) For $2 \leq m \leq n-k$, if

$$
a_{n-r-1}^{(k)} \leq a_{m}^{(k)} \leq a_{n-r}^{(k)},
$$

then

$$
a_{n-r-2}^{(k+1)} \leq a_{m-1}^{(k+1)}, \quad a_{m}^{(k+1)} \leq a_{n-r}^{(k+1)} .
$$

Proof

(i) By Lemma 2.1 we have

$$
\left\{\begin{array}{l}
a_{1}^{(k+1)} \geq a_{2}^{(k+1)} \geq \cdots \geq a_{n-k}^{(k+1)}, \\
a_{n}^{(k+1)} \geq a_{n-1}^{(k+1)} \geq \cdots \geq a_{n-k}^{(k+1)}, \\
a_{1}^{(k+1)} \geq a_{n}^{(k+1)} .
\end{array}\right.
$$


It follows that

$$
a_{1}^{(k+1)}=\max \left\{a_{1}^{(k+1)}, a_{2}^{(k+1)}, \ldots, a_{n}^{(k+1)}\right\} .
$$

Thus we have

$$
a_{n-r}^{(k+1)} \leq a_{1}^{(k+1)}
$$

By the left inequality of (3) we have

$$
a_{2}+\cdots+a_{k+1} \leq a_{n-r}+\cdots+a_{n-r+k-1}
$$

Note that $a_{k+2} \leq a_{k-r}=a_{n+k-r}$, so we have

$$
a_{2}+\cdots+a_{k+1}+a_{k+2} \leq a_{n-r}+\cdots+a_{n-r+k-1}+a_{n+k-r} .
$$

Therefore

$$
a_{2}^{(k+1)} \leq a_{n-r}^{(k+1)}
$$

(ii) By Lemma 2.1 we have

$$
a_{n-k}^{(k+1)} \leq a_{n-r-1}^{(k+1)}
$$

By the right inequality of (4) we get

$$
a_{n-r}+\cdots+a_{n-r+k-1} \leq a_{n-k}+\cdots+a_{n-1} .
$$

Note that $a_{n-r-1} \leq a_{n-k-1}$, so we have

$$
a_{n-r}+\cdots+a_{n-r+k-1}+a_{n-r-1} \leq a_{n-k}+\cdots+a_{n-1}+a_{n-k-1} .
$$

This means that

$$
a_{n-r-1}^{(k+1)} \leq a_{n-k-1}^{(k+1)}
$$

(iii) By (5) we get

$$
a_{m+1}+\cdots+a_{m+k} \leq a_{n-r}+\cdots+a_{n-r+k-1} \leq a_{m}+\cdots+a_{m+k-1} .
$$

Note that $n \geq m+k+1 \geq k-r \geq 1$ and $n \geq n-r-1 \geq m-1 \geq 1$, so we have

$$
a_{m+k+1} \leq a_{n-r+k}=a_{k-r}, \quad a_{n-r-1} \leq a_{m-1} .
$$

It follows that

$$
a_{m+1}+\cdots+a_{m+k}+a_{m+k+1} \leq a_{n-r}+\cdots+a_{n-r+k-1}+a_{n-r+k}
$$


and

$$
a_{n-r-1}+a_{n-r}+\cdots+a_{n-r+k-1} \leq a_{m-1}+a_{m}+\cdots+a_{m+k-1} .
$$

Therefore (6) holds.

(iv) By (7) we get

$$
a_{n}+\cdots+a_{n+k-1} \leq a_{n-k}+\cdots+a_{n-1}
$$

Since $a_{n-1} \leq a_{n-k-1}$, we have

$$
a_{n}+\cdots+a_{n+k-1}+a_{n-1} \leq a_{n-k}+\cdots+a_{n-1}+a_{n-k-1} .
$$

It follows that

$$
a_{n-1}^{(k+1)} \leq a_{n-k-1}^{(k+1)}
$$

(v) By (8) we have

$$
a_{n}+\cdots+a_{n+k-1} \leq a_{m}+\cdots+a_{m+k-1} .
$$

Note that $n-1 \geq m+k$ and $a_{n-1} \leq a_{m+k}$, so we have

$$
a_{n}+\cdots+a_{n+k-1}+a_{n-1} \leq a_{m}+\cdots+a_{m+k-1}+a_{m+k} .
$$

This means that

$$
a_{n-1}^{(k+1)} \leq a_{m}^{(k+1)}
$$

(vi) By (9) we get

$$
a_{n-r-1}+\cdots+a_{n-r+k-2} \leq a_{m}+\cdots+a_{m+k-1} \leq a_{n-r}+\cdots+a_{n-r+k-1} .
$$

Note that $0 \leq r \leq k-2$ and $2 \leq m \leq n-k$, so we have

$$
n \geq n-r-2 \geq n-k \geq m \geq m-1 \geq 1
$$

It follows that

$$
a_{n-r-2} \leq a_{m-1}
$$

So we get

$$
a_{n-r-2}+a_{n-r-1}+\cdots+a_{n-r+k-2} \leq a_{m-1}+a_{m}+\cdots+a_{m+k-1} .
$$

Therefore

$$
a_{n-r-2}^{(k+1)} \leq a_{m-1}^{(k+1)}
$$


Note that $m \leq n-k$, so we have

$$
k-r \leq m+k \leq n, \quad a_{m+k} \leq a_{k-r}=a_{n+k-r} .
$$

It follows that

$$
a_{m}+\cdots+a_{m+k-1}+a_{m+k} \leq a_{n-r}+\cdots+a_{n-r+k-1}+a_{n+k-r} .
$$

Therefore

$$
a_{m}^{(k+1)} \leq a_{n-r}^{(k+1)}
$$

Lemma 2.4 Let $n \geq 4,2 \leq k \leq n-1,1 \leq m \leq n-k$, and $0 \leq r \leq k-2$.

(i) If $a_{m+1}^{(k)} \leq a_{n-r}^{(k)} \leq a_{m}^{(k)}$, then

$$
S_{m+r+1}^{(k)}=\sum_{i=1}^{m} a_{i}^{(k)}+\sum_{i=0}^{r} a_{n-i}^{(k)}
$$

(ii) If $a_{n}^{(k)} \leq a_{m}^{(k)}$, then

$$
S_{m}^{(k)}=\sum_{i=1}^{m} a_{i}^{(k)}
$$

(iii) For $2 \leq m \leq n-k$, if $a_{n-r-1}^{(k)} \leq a_{m}^{(k)} \leq a_{n-r}^{(k)}$, then

$$
S_{m+r+1}^{(k)}=\sum_{i=1}^{m} a_{i}^{(k)}+\sum_{i=0}^{r} a_{n-i}^{(k)}
$$

Proof We only prove (i). Using a similar method, we can obtain (ii) and (iii).

By Lemma 2.1 we have

$$
\left\{\begin{array}{l}
a_{1}^{(k)} \geq a_{2}^{(k)} \geq \cdots \geq a_{m}^{(k)} \geq a_{n-r}^{(k)} \geq a_{m+1}^{(k)} \\
a_{n}^{(k)} \geq a_{n-1}^{(k)} \geq \cdots \geq a_{n-r+1}^{(k)} \geq a_{n-r}^{(k)} \geq a_{m+1}^{(k)}
\end{array}\right.
$$

By Corollary 2.2 we let

$$
S_{m+r+1}^{(k)}=\sum_{i=1}^{h_{1}} a_{i}^{(k)}+\sum_{i=0}^{h_{2}} a_{n-i}^{(k)}
$$

where $1 \leq h_{1} \leq n-k+1,-1 \leq h_{2} \leq k-2$, and $\sum_{i=0}^{-1} a_{n-i}^{(k)}=0$. It is clear that

$$
m+r+1=h_{1}+h_{2}+1 \text {. }
$$

Next, we prove that $h_{1}=m$ and $h_{2}=r$. 
(1) If $h_{1} \geq m+1$, which means that the right-hand side of (12) includes $a_{m+1}^{(k)}$, then by (11) the right-hand side of (12) should include $a_{1}^{(k)}, a_{2}^{(k)}, \ldots, a_{m+1}^{(k)}$ and $a_{n-r}^{(k)}, a_{n-r+1}^{(k)}, \ldots, a_{n}^{(k)}$, so we have

$$
h_{1}+h_{2}+1 \geq m+r+2>m+r+1
$$

This is a contradiction with (13).

(2) If $h_{1} \leq m-1$, then by (13) we have $k-2 \geq h_{2} \geq r+1$. Together with (11), we get

$$
a_{n-h_{2}}^{(k)} \leq a_{n-r-1}^{(k)} \leq a_{n-r}^{(k)} \leq a_{m}^{(k)}
$$

So the right-hand side of (12) must include $a_{m}^{(k)}$, which means that $h_{1} \geq m$. This is a contradiction with $h_{1} \leq m-1$.

Therefore $h_{1}=m$ and $h_{2}=r$. So (10) holds.

Corollary 2.5 Let $n \geq 4,2 \leq k \leq n-2,1 \leq m \leq n-k$, and $0 \leq r \leq k-2$, and let

$$
a_{m+1}^{(k)} \leq a_{n-r}^{(k)} \leq a_{m}^{(k)}
$$

(i) For $m=1$, we have

$$
S_{r+2}^{(k+1)}=a_{1}^{(k+1)}+\sum_{i=0}^{r} a_{n-i}^{(k+1)} .
$$

(ii) For $m=n-k$, we have

$$
S_{n-k+r+1}^{(k+1)}=\sum_{i=1}^{n-k-1} a_{i}^{(k+1)}+\sum_{i=0}^{r+1} a_{n-i}^{(k+1)}
$$

(iii) For $2 \leq k \leq n-3$ and $2 \leq m \leq n-k-1, S_{m+r+1}^{(k+1)}$ must be one of the following two cases:

$$
S_{m+r+1}^{(k+1)}=\sum_{i=1}^{m} a_{i}^{(k+1)}+\sum_{i=0}^{r} a_{n-i}^{(k+1)}
$$

or

$$
S_{m+r+1}^{(k+1)}=\sum_{i=1}^{m-1} a_{i}^{(k+1)}+\sum_{i=0}^{r+1} a_{n-i}^{(k+1)}
$$

Proof

(i) If $m=1$, by (14) we have

$$
a_{2}^{(k)} \leq a_{n-r}^{(k)} \leq a_{1}^{(k)}
$$


By Lemma 2.3(i) we have

$$
a_{2}^{(k+1)} \leq a_{n-r}^{(k+1)} \leq a_{1}^{(k+1)}
$$

and then by Lemma 2.4(i) we can induce that (15) holds.

(ii) If $m=n-k$, then by (14) we have

$$
a_{n-k+1}^{(k)} \leq a_{n-r}^{(k)} \leq a_{n-k}^{(k)}
$$

By Lemma 2.3(ii) we have

$$
a_{n-k}^{(k+1)} \leq a_{n-r-1}^{(k+1)} \leq a_{n-k-1}^{(k+1)}
$$

and then by Lemma 2.4(i) we can induce that (16) holds.

(iii) By Corollary 2.2 we let

$$
S_{m+r+1}^{(k+1)}=\sum_{i=1}^{p} a_{i}^{(k+1)}+\sum_{i=0}^{q} a_{n-i}^{(k+1)},
$$

where $1 \leq p \leq n-k,-1 \leq q \leq k-2$, and $\sum_{i=0}^{-1} a_{n-i}^{(k)}=0$. Then we have

$$
p+q+1=m+r+1
$$

Next, we prove that $p=m$ or $p=m-1$.

(1) If $p \geq m+1$, then by Lemma (2.3)(iii) we have $a_{m+1}^{(k+1)} \leq a_{n-r}^{(k+1)}$. So we get

$$
a_{p}^{(k+1)} \leq a_{m+1}^{(k+1)} \leq a_{n-r}^{(k+1)}
$$

Thus the right-hand side of (17) includes $a_{n-r}^{(k+1)}$, which means that $q \geq r$. Therefore

$$
p+q+1 \geq m+r+2>m+r+1 .
$$

This is a contradiction with (18).

(2) If $1 \leq p \leq m-2$, then by (18) we have $n-q \leq n-r-2$. By Lemma 2.3(iii) we get $a_{n-r-1}^{(k+1)} \leq a_{m-1}^{(k+1)}$. It follows that

$$
a_{n-q}^{(k+1)} \leq a_{n-r-2}^{(k+1)} \leq a_{n-r-1}^{(k+1)} \leq a_{m-1}^{(k+1)} .
$$

So the right-hand side of (17) must include $a_{m-1}^{(k+1)}$. Therefore

$$
p \geq m-1
$$

This is a contradiction with $1 \leq p \leq m-2$.

Thus $p=m$ or $p=m-1$.

In a similar way as in Corollary 2.5, we can prove the following corollaries. 
Corollary 2.6 Let $n \geq 4,2 \leq k \leq n-2,2 \leq m \leq n-k$, and $0 \leq r \leq k-2$.

(i) If $a_{n}^{(k)} \leq a_{m}^{(k)}$, then $S_{m}^{(k+1)}$ must be one of the following two cases:

$$
S_{m}^{(k+1)}=\sum_{i=1}^{m} a_{i}^{(k+1)}
$$

or

$$
S_{m}^{(k+1)}=\sum_{i=1}^{m-1} a_{i}^{(k+1)}+a_{n}^{(k+1)} .
$$

(ii) If $a_{n-r-1}^{(k)} \leq a_{m}^{(k)} \leq a_{n-r}^{(k)}$, then $S_{m+r+1}^{(k+1)}$ must be one of the following two cases:

$$
S_{m+r+1}^{(k+1)}=\sum_{i=1}^{m} a_{i}^{(k+1)}+\sum_{i=0}^{r} a_{n-i}^{(k+1)}
$$

or

$$
S_{m+r+1}^{(k+1)}=\sum_{i=1}^{m-1} a_{i}^{(k+1)}+\sum_{i=0}^{r+1} a_{n-i}^{(k+1)} .
$$

Corollary 2.7 Let $n \geq 4,2 \leq k \leq n-2,1 \leq m \leq n-k$, and $-1 \leq r \leq k-2$, and let $\sum_{i=0}^{-1} a_{n-i}^{(k)}=0$. If

$$
S_{m+r+1}^{(k)}=\sum_{i=1}^{m} a_{i}^{(k)}+\sum_{i=0}^{r} a_{n-i}^{(k)},
$$

then we have:

(i) if $m=1$, then

$$
S_{r+2}^{(k+1)}=a_{1}^{(k+1)}+\sum_{i=0}^{r} a_{n-i}^{(k+1)} ;
$$

(ii) if $2 \leq m \leq n-k$, then $S_{m+r+1}^{(k+1)}$ must be one of the following two cases:

$$
S_{m+r+1}^{(k+1)}=\sum_{i=1}^{m} a_{i}^{(k+1)}+\sum_{i=0}^{r} a_{n-i}^{(k+1)}
$$

or

$$
S_{m+r+1}^{(k+1)}=\sum_{i=1}^{m-1} a_{i}^{(k+1)}+\sum_{i=0}^{r+1} a_{n-i}^{(k+1)} .
$$

Lemma 2.8 Let $n \geq 4,2 \leq k \leq n-2,1 \leq m \leq n-k$, and $-1 \leq r \leq k-2$, and let $\sum_{i=0}^{-1} a_{n-i}^{(k)}=0$. If

$$
\left\{\begin{array}{l}
S_{m+r+1}^{(k)}=\sum_{i=1}^{m} a_{i}^{(k)}+\sum_{i=0}^{r} a_{n-i}^{(k)}, \\
S_{m+r+1}^{(k+1)}=\sum_{i=1}^{m} a_{i}^{(k+1)}+\sum_{i=0}^{r} a_{n-i}^{(k+1)},
\end{array}\right.
$$


then

$$
S_{m+r+1}^{(k)} \geq S_{m+r+1}^{(k+1)}
$$

Proof By a simple calculation we obtain

$$
S_{m+r+1}^{(k)}-S_{m+r+1}^{(k+1)}=\frac{1}{k+1}\left(S_{m+r+1}^{(k)}-\sum_{i=k-r}^{k+m} a_{i}\right)
$$

By (19) we have

$$
a_{m+1}^{(k)} \leq a_{n-r}^{(k)}
$$

It follows that

$$
a_{m+1}+a_{m+2}+\cdots+a_{k+m} \leq a_{n-r}+a_{n-r+1}+\cdots+a_{n-r+k-1} .
$$

So we have

$$
a_{k-r}+a_{k-r+1}+\cdots+a_{k+m} \leq a_{n-r}+a_{n-r+1}+\cdots+a_{n+m} .
$$

Note that

$$
\begin{cases}a_{n-r}+a_{n-r+1}+\cdots+a_{n+m} \leq a_{n-r+j}+a_{n-r+1+j}+\cdots+a_{n+m+j}, & 0 \leq j \leq r \\ a_{k-r}+a_{k-r+1}+\cdots+a_{k+m} \leq a_{n-r+j}+a_{n-r+1+j}+\cdots+a_{n+m+j}, & r+1 \leq j \leq k-1 .\end{cases}
$$

Thus we can induce that

$$
S_{m+r+1}^{(k)}=\sum_{i=1}^{m} a_{i}^{(k)}+\sum_{i=0}^{r} a_{n-i}^{(k)}=\frac{1}{k} \sum_{i=n-r}^{n+m} \sum_{j=0}^{k-1} a_{j+i}=\frac{1}{k} \sum_{j=0}^{k-1} \sum_{i=n-r}^{n+m} a_{j+i} \geq \sum_{i=k-r}^{k+m} a_{i}
$$

This means that (20) holds.

Lemma 2.9 Let $n \geq 4,2 \leq k \leq n-2,2 \leq m \leq n-k$, and $-1 \leq r \leq k-2$, and let $\sum_{i=0}^{-1} a_{n-i}^{(k)}=0$. If

$$
\left\{\begin{array}{l}
S_{m+r+1}^{(k)}=\sum_{i=1}^{m} a_{i}^{(k)}+\sum_{i=0}^{r} a_{n-i}^{(k)} \\
S_{m+r+1}^{(k+1)}=\sum_{i=1}^{m-1} a_{i}^{(k+1)}+\sum_{i=0}^{r+1} a_{n-i}^{(k+1)}
\end{array}\right.
$$

then

$$
S_{m+r+1}^{(k)} \geq S_{m+r+1}^{(k+1)}
$$

Proof Note that

$$
S_{m+r+1}^{(k)}-S_{m+r+1}^{(k+1)}
$$




$$
\begin{aligned}
& =\frac{1}{k+1}\left(\sum_{i=1}^{m-1} a_{i}^{(k)}+\sum_{i=0}^{r} a_{n-i}^{(k)}\right)+a_{m}^{(k)}-a_{n-r-1}^{(k+1)}-\frac{1}{k+1} \sum_{i=k-r}^{k+m-1} a_{i} \\
& =\frac{1}{k+1}\left(\sum_{i=1}^{m} a_{i}^{(k)}+\sum_{i=0}^{r} a_{n-i}^{(k)}-\sum_{i=n-r-1}^{n+m-1} a_{i}\right) \\
& =\frac{1}{k+1}\left(S_{m+r+1}^{(k)}-\sum_{i=n-r-1}^{n+m-1} a_{i}\right) .
\end{aligned}
$$

By (21) we have

$$
a_{n-r-1}^{(k)} \leq a_{m}^{(k)}
$$

It follows that

$$
a_{n-r-1}+a_{n-r}+\cdots+a_{n-r+k-2} \leq a_{m}+a_{m+1}+\cdots+a_{m+k-1} \text {. }
$$

So we can induce that

$$
a_{n-r-1}+a_{n-r}+\cdots+a_{n+m-1} \leq a_{k-r-1}+a_{k-r}+\cdots+a_{k+m-1} .
$$

Since

$$
\begin{cases}a_{n-r-1}+a_{n-r}+\cdots+a_{n+m-1} \leq a_{n-r+j}+a_{n-r+1+j}+\cdots+a_{n+m+j}, & 0 \leq j \leq r, \\ a_{k-r-1}+a_{k-r}+\cdots+a_{k+m-1} \leq a_{n-r+j}+a_{n-r+1+j}+\cdots+a_{n+m+j}, & r+1 \leq j \leq k-1,\end{cases}
$$

we have

$$
S_{m+r+1}^{(k)}=\frac{1}{k} \sum_{j=0}^{k-1} \sum_{i=n-r}^{n+m} a_{j+i} \geq \sum_{i=n-r-1}^{n+m-1} a_{i} .
$$

This means that (22) holds.

\section{Main results}

We are now in a position to prove our main results (1) in two cases: $k=1$ and $2 \leq k \leq n-1$.

Theorem 3.1 For any $n \geq 2$, we have

$$
a^{(2)} \prec a^{(1)}=a .
$$

Proof It is clear that (23) holds if $n=2$. Next, let $n \geq 3$. Then we have

$$
\frac{a_{1}+a_{2}}{2}=S_{1}^{(2)} \leq S_{1}^{(1)}=a_{1}, \quad S_{n}^{(2)}=S_{n}^{(1)} .
$$

For $2 \leq m \leq n-1$, we prove that $S_{m}^{(2)} \leq S_{m}^{(1)}$ in the following two cases:

(i) If $S_{m}^{(2)}=\sum_{i=1}^{m} a_{i}^{(2)}$, then

$$
S_{m}^{(2)}-S_{m}^{(1)}=\frac{a_{m+1}-a_{1}}{2} \leq 0 .
$$


(ii) If $S_{m}^{(2)}=a_{n}^{(2)}+\sum_{i=1}^{m-1} a_{i}^{(2)}$, then

$$
S_{m}^{(2)}-S_{m}^{(1)}=\frac{a_{n}-a_{m}}{2} \leq 0 .
$$

So (23) holds.

Theorem 3.2 For any $n \geq 3$ and $2 \leq k \leq n-1$, we have

$$
a^{(k+1)} \prec a^{(k)} .
$$

Proof It is clear that (24) holds for any $n \geq 3, k=n-1$ and for $n=3, k=1$.

Next, let $n \geq 4$ and $2 \leq k \leq n-2$.

For any $1 \leq m \leq n-k$ and $-1 \leq r \leq k-2$, let $\sum_{i=0}^{-1} a_{n-i}^{(k)}=0$, and let

$$
S_{m+r+1}^{(k)}=\sum_{i=1}^{m} a_{i}^{(k)}+\sum_{i=0}^{r} a_{n-i}^{(k)} .
$$

Next, we prove that

$$
S_{m+r+1}^{(k)} \geq S_{m+r+1}^{(k+1)}
$$

in the following two cases:

(i) If $m=1$ and $-1 \leq r \leq k-2$, then by Corollary $2.7(\mathrm{i})$ and Lemma 2.8 we get

$$
S_{r+2}^{(k)} \geq S_{r+2}^{(k+1)} .
$$

(ii) If $2 \leq m \leq n-k$ and $-1 \leq r \leq k-2$, then by Corollary 2.7(ii), Lemma 2.8, and Lemma 2.9 we get

$$
S_{m+r+1}^{(k)} \geq S_{m+r+1}^{(k+1)}
$$

Note that

$$
S_{n}^{(k)}=S_{n}^{(k+1)}
$$

so (24) holds.

\section{Discussion}

In the theory of majorizations, there are two key concepts, majorizing relations and Schurconvex functions. Majorizing relations are weaker ordered relations among vectors, and Shur-convex functions are an extension of classical convex functions. Combining these two objects is an effective method of constructing inequalities.

In the theory of majorization, there are two important and fundamental objects, establishing majorizing relations among vectors and finding various Schur-convex functions. Majorizing relations deeply characterize intrinsic connections among vectors, and combining a new majorizing relation with suitable Schur-convex functions can lead to various interesting inequalities; see [3-13]. 


\section{Funding}

This research was supported partially by the Natural Science Foundation of China under Grant $(11371185,11761029$,

11361038).

\section{Competing interests}

The authors declare that they have no competing interests.

\section{Authors' contributions}

All authors contributed equally and significantly in this paper. All authors read and approved the final manuscript.

\section{Author details}

${ }^{1}$ School of Mathematical Sciences, Inner Mongolia University, Hohhot, China. ${ }^{2}$ Teacher's College, Beijing Union University, Beijing, China. ${ }^{3}$ College of Mathematics, Inner Mongolia University for Nationalities, Tongliao, China.

\section{Publisher's Note}

Springer Nature remains neutral with regard to jurisdictional claims in published maps and institutional affiliations.

Received: 18 January 2018 Accepted: 16 June 2018 Published online: 28 June 2018

\section{References}

1. Marshall, A.W., Olkin, I., Arnord, B.C.: Inequalities: Theory of Majorization and Its Application, 2nd edn. Springer, New York (2011)

2. Shi, H.N.: A class of majorization inequality with applications. J. Beijing Union Univ. (Nat. Sci.) 24(1), 60-64 (2010)

3. Wu, S.H., Shi, H.N.: A relation of weak majorization and its applications to certain inequalities for means. Math. Slovaca 61(4), 561-570 (2011)

4. Shi, H.N., Jiang, Y.M., Jiang, W.D.: Schur-convexity and Schur-geometrically concavity of Gini means. Comput. Math. Appl. 57, 266-274 (2009)

5. Shi, H.N., Wu, S.H., Qi, F.: An alternative note on the Schur-convexity of the extended mean values. Math. Inequal. Appl. 9, 219-224 (2006)

6. Shi, H.N.: Schur-convex functions related to Hadamard-type inequalities. J. Math. Inequal. 1, 127-136 (2007)

7. Shi, H.N., Zhang, J.: Compositions involving Schur geometrically convex functions. J. Inequal. Appl. 2015, 320 (2015). https://doi.org/10.1186/s13660-015-0842-x

8. Shi, H.N., Zhang, J.: Compositions involving Schur harmonically convex functions. J. Comput. Anal. Appl. 22(5), 907-922 (2017)

9. Shi, H.N., Zhang, J.: Some new judgment theorems of Schur geometric and Schur harmonic convexities for a class of symmetric functions. J. Inequal. Appl. 2013, 527 (2013). https://doi.org/10.1186/1029-242X-2013-527

10. Shi, H.N., Zhang, J.: Schur-convexity of dual form of some symmetric functions. J. Inequal. Appl. 2013, 295 (2013). https://doi.org/10.1186/1029-242X-2013-295

11. Shi, H.N., Zhang, J.: Schur-convexity, Schur-geometric and harmonic convexities of dual form of a class symmetric functions. J. Math. Inequal. 8(2), 349-358 (2014)

12. Shi, H.N., Zhang, J., Ma, Q.H.: Schur-convexity, Schur-geometric and Schur-harmonic convexity for a composite function of complete symmetric function. SpringerPlus 5, 296 (2016). https://doi.org/10.1186/s40064-016-1940-z

13. Shi, H.N., Li, D.M., Gu, C.: Schur-convexity of a mean of convex function. Appl. Math. Lett. 22(6), 932-937 (2009)

\section{Submit your manuscript to a SpringerOpen ${ }^{\circ}$ journal and benefit from:}

- Convenient online submission

- Rigorous peer review

- Open access: articles freely available online

- High visibility within the field

- Retaining the copyright to your article

Submit your next manuscript at $\boldsymbol{\Delta}$ springeropen.com 